\title{
Unnecessary shock from an implantable cardioverter- defibrillator following transcutaneous pacing
}

\author{
Riyaz Somani, PhD, MRCP; Peggy DeJong, MD; Kevin Michael, MB ChB; \\ Adrian Baranchuk, MD, FACC
}

\section{ABSTRACT}

As the population ages and cardiovascular disease becomes more prevalent, an increasing number of patients are receiving implantable cardioverter-defibrillators (ICDs). When these patients present to the emergency department, it is imperative that physicians are not only aware of the possible underlying medical issues that may have precipitated their admission but should also have a good understanding of the potential interactions that any medical intervention may have on the patient's device. We discuss a case in which a patient known to have an ICD in situ was transcutaneously paced for the management of bradycardia, leading to an unnecessary shock.

\section{RÉSUMÉ}

À mesure que la population vieillit et que la fréquence des maladies cardiovasculaires augmente, de plus en plus de patients portent un défibrillateur automatique implantable (DAI). Lorsque ces patients vont consulter au service des urgences, il est impératif que les médecins non seulement connaissent les problèmes médicaux sous-jacents qui peuvent avoir précipité leur admission, mais qu'ils aient aussi une bonne compréhension des interactions possibles que toute intervention médicale peut avoir sur l'appareil. II sera ici question du cas d'un patient connu pour être porteur d'un DAl, qui a subi une stimulation transcutanée pour de la bradycardie, mais qui, par suite de l'intervention, a reçu inutilement un choc.

Keywords: emergency department, implantable cardioverter defibrillator, transcutaneous pacing, unnecessary shock

\section{CASE REPORT}

A 66-year-old man with ischemic cardiomyopathy (ejection fraction 19\%) underwent implantation of a single-chamber implantable cardioverter-defibrillator (ICD) (Virtuoso VR, Medtronic, Minneapolis, MN) in 2007 for primary prevention of sudden cardiac death. In a bid to minimize right ventricular pacing (which is recognized to be detrimental), the bradycardia parameters of his device were programmed to VVI 40 beats/min to ensure that ventricular pacing would occur only if his intrinsic heart rate fell below this level. Given that the device was implanted on primary prevention grounds (with no previously documented ventricular tachycardia $[\mathrm{VT}]$ or fibrillation $[\mathrm{VF}]$ ), it was programmed with a single therapy zone for VF set at $\geq 187.5$ beats $/ \mathrm{min}$. Thus, a heart rate exceeding this would result in the delivery of $35 \mathrm{~J}$ shocks.

The patient presented to hospital following a syncopal episode and was found to be bradycardic, receiving VVI pacing at 40 beats/min (Figure 1), with a blood pressure of $70 / 44 \mathrm{~mm} \mathrm{Hg}$. While awaiting the arrival of an electrophysiologist, in addition to commencing inotropes, the emergency physicians attempted to transcutaneously pace the patient (Lifepak 12 , Medtronic) at a fixed rate of 100 beats $/ \mathrm{min}$. The patient subsequently had a pulseless electrical activity (PEA) cardiac arrest that necessitated cardiopulmonary resuscitation (CPR). The patient required intubation and ventilation before being transferred to the intensive care unit for ongoing supportive care. The patient was extubated 2 days later and was subsequently discharged home. The cause of the patient's initial syncopal episode and bradycardia were later deemed to be drug induced (a $\beta$-blocker and amiodarone).

Interrogation of the ICD revealed one detected episode that the device had interpreted as VF, the

From the Heart Rhythm Service, Kingston General Hospital, Queen's University, Kingston, ON.

Correspondence to: Dr. Riyaz Somani, Heart Rhythm Service, Kingston General Hospital, Queen's University, Kingston, ON K7L 2V7; riyazsomani@ hotmail.com.

This article has been peer reviewed. 


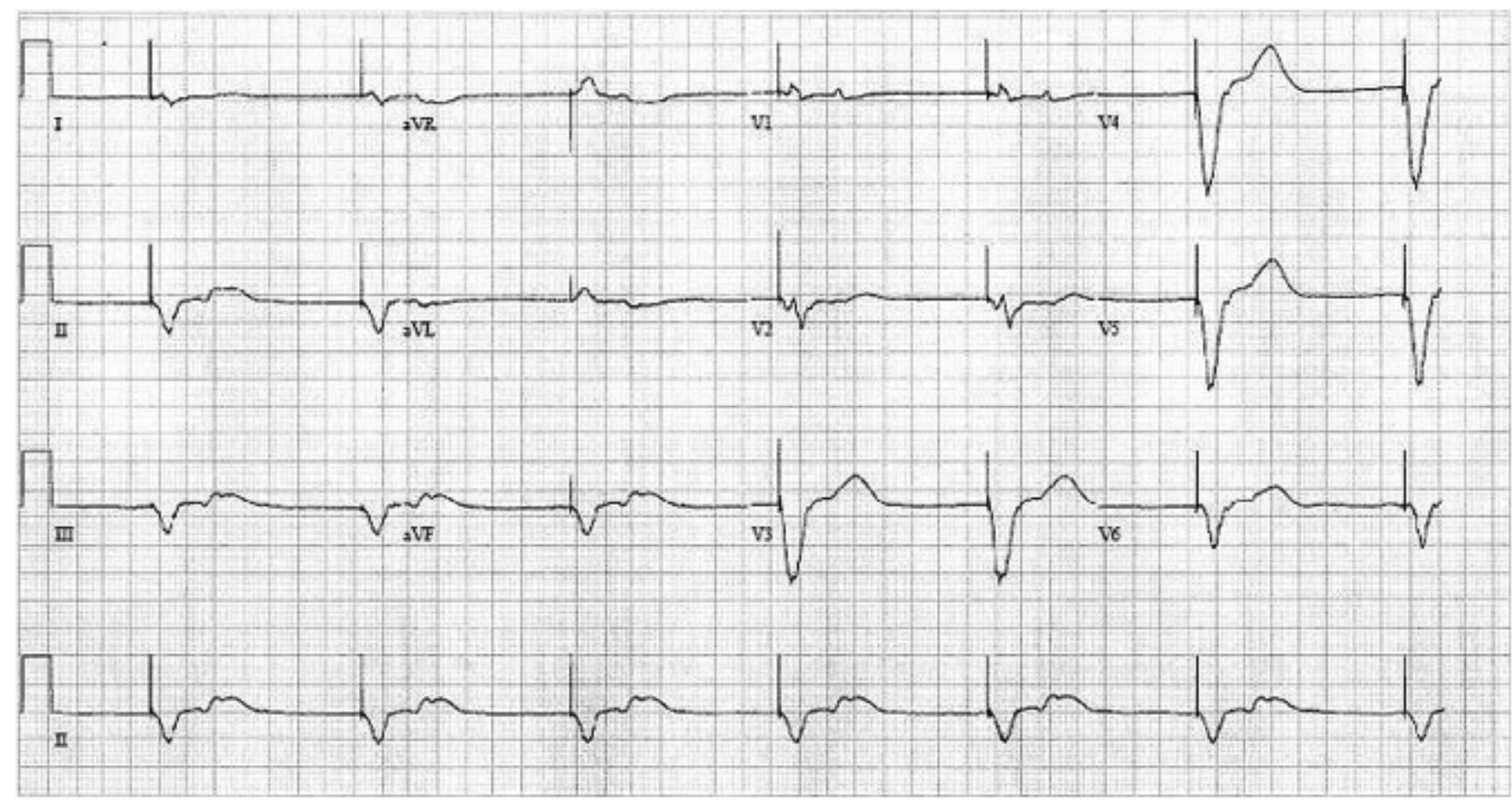

Figure 1. A 12-lead electrocardiogram of the patient's presenting rhythm showing right ventricular pacing at 40 beats/min.

timing of which corresponded to shortly after commencement of transcutaneous pacing. The dot-plot (Figure 2A) initially revealed a scattering of $\mathrm{V}-\mathrm{V}$ intervals, some of which were in the $\mathrm{VF}$ detection zone, before delivery of a $34.7 \mathrm{~J}$ shock. Inspection of the corresponding electrograms (Figure 2B) revealed an intrinsic heart rate of approximately 150 beats $/ \mathrm{min}$. Superimposed on this were pacing spikes (from transcutaneous pacing), which, although failed to capture (no evidence of ventricular depolarization following pacing spikes), resulted in the device interpreting these as short $\mathrm{V}-\mathrm{V}$ intervals and declaring $\mathrm{VF}$. Following delivery of a shock, the intrinsic heart rate slowed (see Figure 2B) to approximately 75 beats/min, with transcutaneous pacing spikes still evident.

\section{DISCUSSION}

With the dramatic increase in the number of pacemaker and ICD implantations over the last 10 years, it is vital that emergency physicians have some understanding of the potential reasons that these patients may present. Although uncommon, rhythm complications include failure to pace (due to oversensing, battery depletion, or lead displacement/fracture), undersensing (resulting in inappropriate pacing), pacemaker-mediated tachycardia, and inappropriate shocks from ICDs (due to supraventricular tachycardias or problems with lead integrity).

This particular case highlights the potential complications that may arise when patients with ICDs are seen by physicians unfamiliar with these devices. Interrogation of the device confirmed that the ICD was functioning appropriately throughout, pacing the heart when the intrinsic rhythm fell below 40 beats/min and delivering a shock when it perceived the heart rate to be in excess of 187.5 beats/min. However, the use of transcutaneous pacing in this patient with an existing ICD, together with the unrecognized resolution of bradycardia, led to an unnecessary shock from the device. It is likely that the patient's subsequent PEA arrest was related to myocardial stunning, precipitated by the ICD shock in the presence of pre-existing severely impaired left ventricular systolic function and cardiac hypoperfusion secondary to bradycardia., ${ }^{1,2}$ Although the patient did make a full recovery after a period of hemodynamic and ventilatory support, the outcome could potentially have been disastrous, particularly if the shock had been delivered during the vulnerable repolarizing portion of the cardiac cycle, which may have resulted in the induction of $\mathrm{VF}^{3}$

Transcutaneous pacing provides a rapid, noninvasive means of causing cardiac depolarization resulting in 


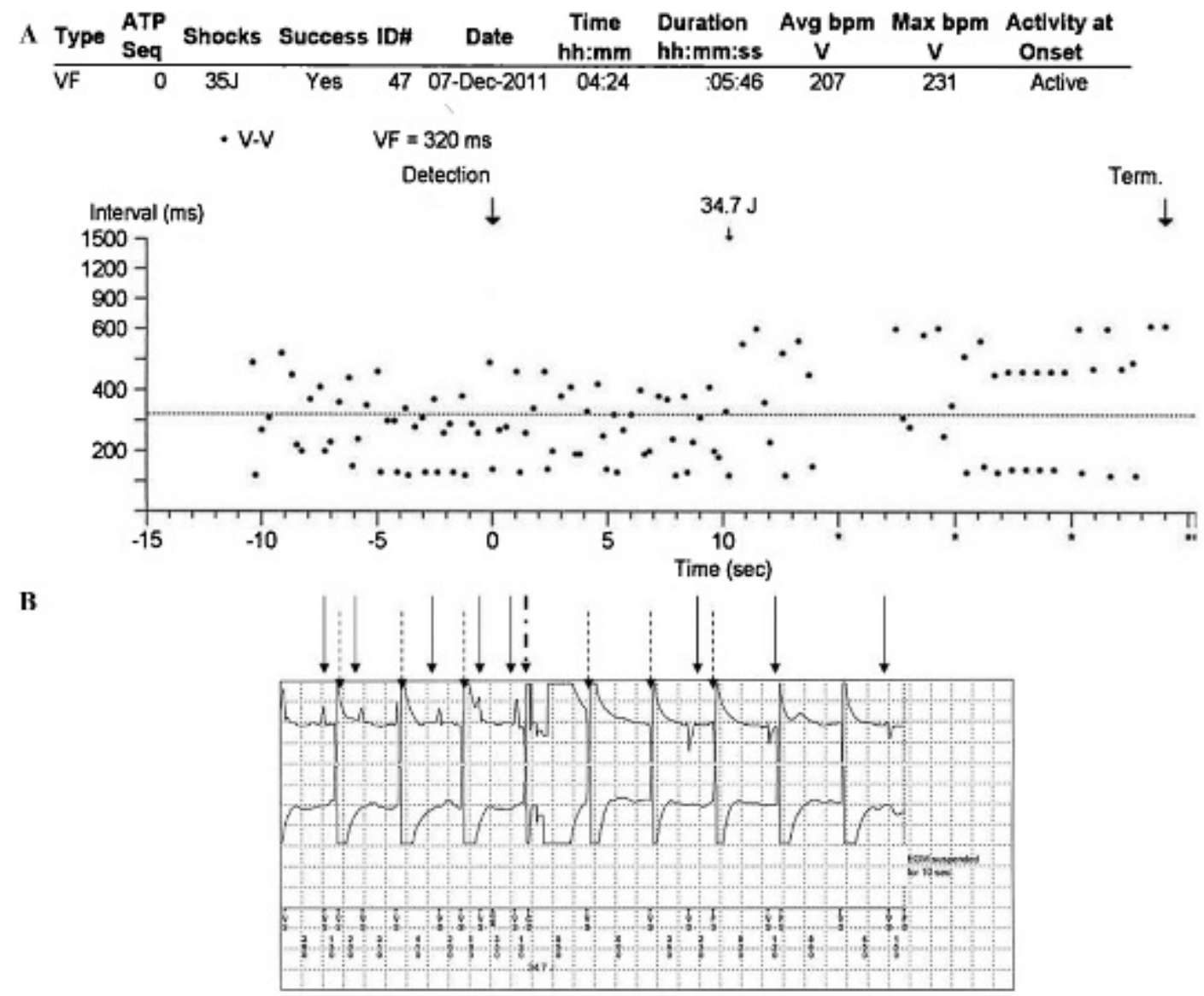

Figure 2. $A, A$ dot-plot showing the event during which time fixed-rate transcutaneous pacing was switched on. There is a wide scattering of $\mathrm{V}-\mathrm{V}$ intervals, a significant number of which fall below the ventricular fibrillation (VF) detection line of 320 ms (equivalent to 187.5 beats/min). VF is subsequently declared, and a $34.7 \mathrm{~J}$ shock is administered. The varied V-V intervals initially persist before the device declares the episode terminated. $B$, The near-field electrogram (RVtip-RVring) during transcutaneous pacing shows an intrinsic heart rate at 150 beats/min (solid arrows) interspersed by spikes generated through transcutaneous pacing (dashed arrows). The resultant short $\mathrm{V}-\mathrm{V}$ intervals lead to a $34.7 \mathrm{~J}$ shock (dash-dot arrow) being delivered, resulting in slowing of the intrinsic heart rate to approximately 75 beats/min (solid arrows), and likely represents cardioversion of an atrial flutter or an atrial tachycardia to sinus rhythm. Transcutaneous pacing spikes continue following the shock. ATP = antitachycardia pacing.

myocardial contraction and is often employed as a means of treating bradycardia in the emergency department. Although when transcutaneous pacing was initially commenced in this patient he was presumably bradycardic, interrogation of the ICD suggests that immediately prior to delivery of the shock, the patient had an intrinsic heart rate of 150 beats/min, during which time attempts at transcutaneous pacing persisted. The combination of the patient's intrinsic heart rate, together with the pacing spikes generated by the transcutaneous pacing, resulted in short $\mathrm{V}-\mathrm{V}$ intervals being detected by the ICD, resulting in an unnecessary shock. Although in general the use of transcutaneous pacing would not routinely be favoured in a patient with an existing ICD, it may be undertaken safely in an emergency situation provided that ICD therapies are first deactivated with the use of a magnet being placed over the ICD generator. This would allow the safe use of transcutaneous pacing in a patient with an ICD without the risk of precipitating an unnecessary shock. ${ }^{4}$ Alternatively, the use of one of the newer generation of transcutaneous pacemakers, which has the capability of sensing intrinsic ventricular activity and therefore delivers a pacing spike only when the intrinsic heart rate falls below the programmed rate (if available), may also prevent an unnecessary shock.

Given the sequence of events, we would speculate that in addition to failing to recognize the recovery of an intrinsic rhythm, the possibility of an 
interaction between fixed-rate transcutaneous pacing and the ICD and the potential for precipitating a shock from the ICD had not been considered. Delivery of a shock in this patient did, however, result in restoration of sinus rhythm with a heart rate of 75 beats/min (potentially cardioverting an atrial flutter or an atrial tachycardia at a rate of 150 beats/ min) (see Figure 2B).

\section{CONCLUSION}

This case illustrates an iatrogenic cause for an ICD shock precipitated by the use of transcutaneous pacing in a patient with an ICD. Given the possible proarrhythmic effects in patients with ICDs, transcutaneous pacing should ideally be avoided, but if deemed essential, then appropriate steps should be undertaken to deactivate ICD therapies (with a magnet) prior to its use, or by using transcutaneous pacemakers, which have sensing capabilities.

Competing interests: None declared.

\section{REFERENCES}

1. Tokano T, Bach D, Chang J, et al. Effect of ventricular shock strength on cardiac hemodynamics. 7 Cardiovasc Electrophysiol 1998;9:791-7, doi:10.1111/j.1540-8167.1998.tb00118.x.

2. Yamaguchi H, Weil M, Tang W, et al. Myocardial dysfunction after electrical defibrillation. Resuscitation 2002;54:289-96, doi:10.1016/S0300-9572(02)00149-1.

3. Tung R, Zimetbaum P, Josephson ME. A critical appraisal of implantable cardioverter-defibrillator therapy for the prevention of sudden cardiac death. 7 Am Coll Cardiol 2008;52:111121, doi:10.1016/j.jacc.2008.05.058.

4. Jacob S, Panaich SS, Maheshwari R, et al. Clinical applications of magnets on cardiac rhythm management devices. Europace 2011;13:1222-30, doi:10.1093/europace/eur137. 